\title{
Exploring Film Failure Mechanism and Scratch Toughness Characterization of Diamond-Like Carbon Film
}

\author{
Wang Yonghui \\ China University of Geosciences (Beijing) \\ Beijing, China
}

\author{
Yu Xiang* \\ China University of Geosciences (Beijing) \\ Beijing, China \\ e-mail: yuxiang@cugb.edu.cn
}

\begin{abstract}
Diamond-like carbon (DLC) film has drawn an intensive concern, but suffers from its poor toughness. Characterization method for the film toughness is not available to date, which hampers the researches for toughening DLC films. This work attempts to reveal the scratch failure mechanism from stress aspects, and then allows for a characterization method to determine the toughness with accuracy. Results show that, (a) failure process of the DLC film under increasing load during scratch duration can be divided into three stages: (I) circular cracks occurred on the upper surface; (II) radial cracks formed in the lower surface; and (III) lateral cracks appeared in the film due to crack converging, leading to delamination and spalling of the film. (b) Scratch morphology allows for a qualitative characterization; as for the film toughness unavailable from the scratch morphology, the value of Scratch Crack Propagation Resistance (CPR $)$ can quantitatively characterize the film toughness.
\end{abstract}

Keywords-diamond-like carbon (DLC) film; toughness characterization; scratch test; failure mechanism; stress aspect

\section{INTRODUCTION}

DLC (diamond-like carbon) film has a strong application potential in green precision manufacturing industry due to its high hardness, smooth surface, and good corrosion resistance. However, DLC film subjects to a poor toughness as hard film ${ }^{[1]}$, and its failure mode is abrupt catastrophic delamination in majority, which seriously affects its tribological application prospects. Its failure is primarily caused by high internal stress accumulated during synthesis of diamond phase in carbon valence bond network ${ }^{[2]}$. Two main methods to improve the film toughness are: (i) preparing a metal transition layer between the film and the substrate; (ii) doping metal into the DLC film.

In essence, toughness means the energy absorption capacity of a film material during the transition from deformation to fracture. It is an important mechanical property relating to the cohesive force of DLC film, and reflects the film capacity to resist the formation of cracks near defects in the film due to stress accumulation. A method that can characterize the toughness of DLC films is still an unresolved issue in the way of investigating how to toughen these films. As thicknesses of DLC films are in micron magnitude in majority, it is impossible to characterize toughness of DLC films using traditional methods. Our previous results ${ }^{[3-5]}$ show that, (i) the adhesion between DLC film and substrate can be improved by applying metal interface layer and metal doping; (ii) the adhesion is closely related to the toughness of the substrate and the film. Unfortunately, our previous study lacks an understanding of the failure mechanism of the film adhesion as well as an accurate toughness characterization method.

Due to convenience and effectiveness, the scratch test is a preferred method to characterize the toughness of DLC films. The traditional characterization ${ }^{[6]}$ method for the toughness is using the initial (first) critical load $\left(\mathrm{L}_{c 1}\right)$ in scratch test to evaluate the ability of film to resist crack initiation and propagation. Our results demonstrated some exceptions. A typical example is, in case a film owns a good resistance to crack propagation, such film may not lapse immediately after approaching $\mathrm{L}_{c 1}$, but can endure for certain duration before its failure. In light of these findings, this study attempts to reveal the scratch failure mechanism, and then to explore a characterization method to determine the toughness of DLC films with accuracy.

\section{SCRATCH FAILURE MECHANISM}

\section{A. Scratch morphology}

We conducted scratch tests on some DLC films doped with different contents of Ag nanocrystallites [3], and whose scratch morphologies are shown in Fig.1.

As shown in Fig.1a, b, and f, for the DLC films doped with a relatively low metal content or an excessive metal content, there are many obvious cracks on the surface of scratch trace and some spalling around the scratch trace. This indicates that these films had poor resistance crack extension ability. On the other hand, there are several cracks on the scratch trace surface in Fig.1c and e, and few spalling occurs approaching the critical load. This indicates that the films have a certain ability to resist crack extension, but cannot resist the crack extension at high load. As for the film shown in Fig.1d, there is almost no crack on the scratch surface, and the scratch surface has a smooth adjacency, which implies good resistance for crack extension.

We can see from Fig. 1 that, the film exhibits different scratch morphologies under the same experimental condition. A variety of scratch morphologies correlate with varying crack extension resistance of the film around the 
indenter tip. This means that the scratch morphology has a close relationship with the stress state of the film around the indenter tip. In this way, we attempt to explore the failure mechanism of scratch test from stress aspect, by means of formation process of the cracks.
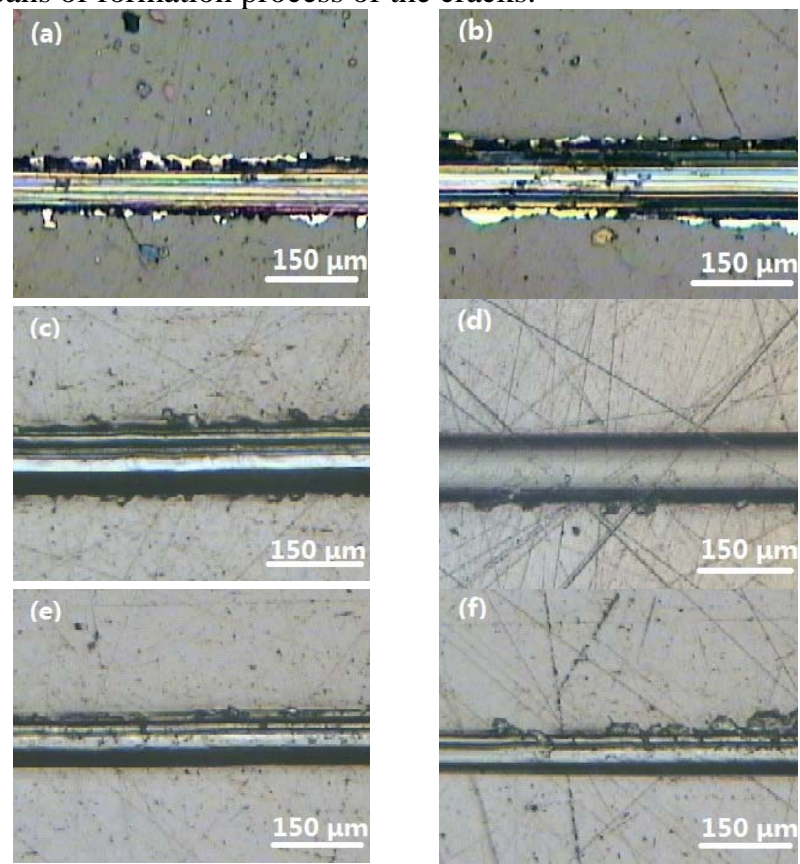

Figure 1. Morphology images of the failure regions on the surfaces of the Ag-DLC films resulting from the scratch test: (a) a:C-Ag0\%, (b) a:CAg5.3\%, (c) a:C-Ag9.3\%, (d) a:C-Ag15.2\%, (e) a:C-Ag24.6\% and (f) a:C-Ag31.8\%.

\section{B. Failure mechanism of scratch test}

During the scratch test, a scratch trace on the film surface is produced by indenter. The load on the indenter tip causes a shear stress in the indentation region edge, which is directly proportional to the applying load. In addition, the film before the indenter tip endures compressive stress, whereas the film behind the tip endures tensile stress. The tensile stress may lead to the cohesive and adhesive failures of the film, which are manifested by

crack initiation and extension or the separation between the film and the substrate.

Fig. 2 shows the possible mechanism of crack initiation and extension in a Me (metal)-DLC film during scratch test. With load increasing, the failure process of the film can be divided into three stages as follows.

(I) Circular cracks are initially formed behind the indenter (Fig.2-I). The friction on the film increases as the load increases, i.e., the tensile stress on the film behind the indenter increases with increasing load. Cracks initiate when the load reaches $\mathrm{L}_{c 1}$, and extend to form circular cracks paralleling with the indenter.

(II) Radial cracks are formed at the interface between the film and substrate (Fig.2-II). The film bends due to the vertical pressure from the indenter. The upper surface of the film endures a compressive stress, whereas the lower surface of the film endures a tensile stress. Thus radial cracks vertical to the direction of the scratch are formed at the interface between the film and substrate.

(III) As the applied pressure increases, the circular cracks encounter the radial cracks, and Fig.2-III shows the profile of the test sample. These two kinds of cracks further extend as the applied pressure continually increases. These two types of cracks converge to form lateral cracks, resulting in the delamination of the film from the substrate.

Based on above analysis of the stress statuses on the film, we can see that micro-cracks will initiate under tensile stress, and then extend and converge, resulting in the film delaminate from the substrate The film failure mechanism correlates with evolution of the three stages, (I) circular cracks formed, (II) radial cracks formed, and (III) lateral cracks formed due to crack converging. The ability difference of crack initiation and extension resistance leads to different statuses of the three stages, and thus result in a variety of scratch morphologies.

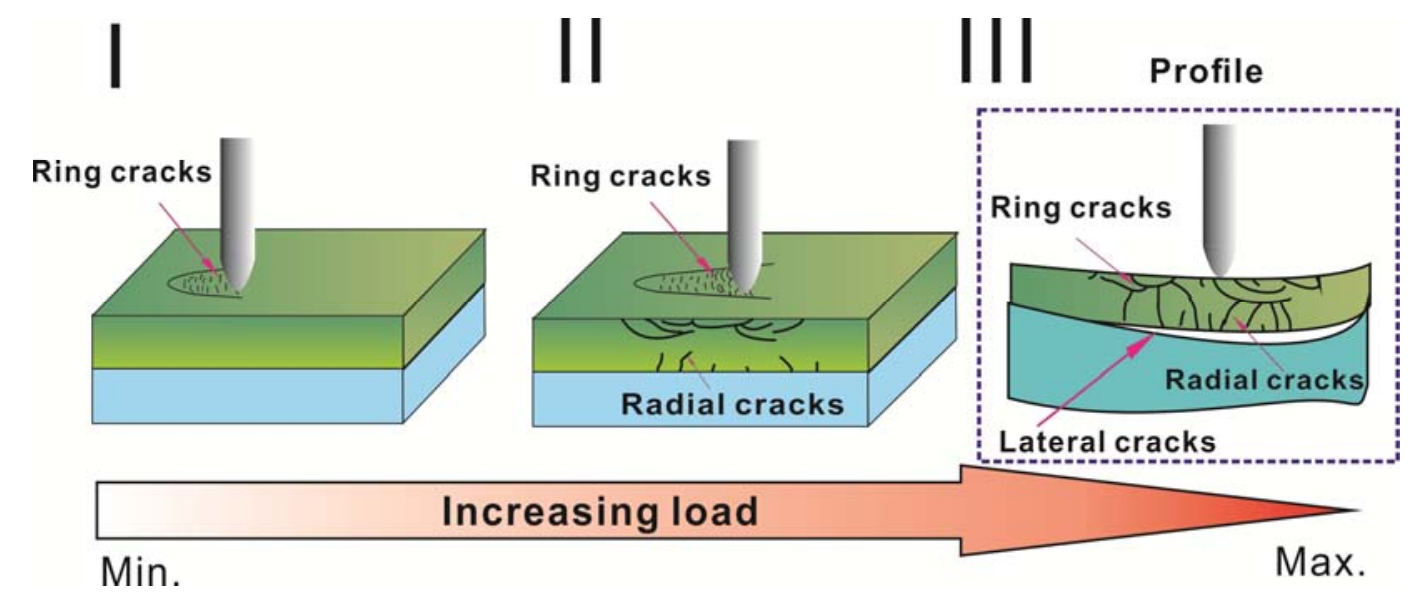

Figure 2. Possible mechanism of crack initiation and extension in a Me-DLC film during scratch test 


\section{CHARACTERIZE FILM TOUGHNESS BY SCRATCH TEST}

\section{A. Qualitative Characterization of Film Toughness}

We can see from the above failure mechanism that the tensile stress and shear stress may induce the crack initiation and extension, or even the spalling from the substrate. The film evolves gradually from stage I to stage III under an increasing applied load. A poor crack initiation and extension resistance will facilitate evolution of the circular and radial cracks during stages I-III; otherwise, the cracks will difficult to initiate and extend, even result in part absence of the three stages. Toughness reflects the ability of the film to resist the initiation and extension of cracks, and is caused by stress accumulation around defects, i.e., toughness is proportional to resistance ability of crack initiation and extension. In this way, the film toughness can be qualitatively characterized through analyzing the crack distribution from stress aspect.

A close observation of the scratch morphologies in Fig.1 lets us know that: (i) The films in Fig.1a, b, and $f$ own a poor toughness, since rich delamination and spalling around the scratch trace illustrate a full evolution of the circular and radial cracks progressed during stages I-III. (ii) The films in Fig.1c and e own a better toughness than that of Fig.1a, b, and f, since the fewer cracks and spalling around the scratch trace imply an unfavorable evolution of the cracks at stage II and III. (iii) The film in Fig.1d owns an optimal toughness. In that almost no cracks and spalling can be seen in the scratch surface, leaving a smooth surround. The scratch morphology indicates that the crack was hard to initiate at stage I, and resulted in the absence of stage II and III.

\section{B. Quantitative Characterization of Film Toughness}

1) Principle of quantitatively characterization of toughness

The quantitatively characterization needs to use in case that the convenient qualitative characterization cannot determine the scratch toughness. Using the films in Fig.1a, b, and $\mathrm{f}$ as example, if difference of the films scratch morphology is not much, the qualitative characterization cannot determine whose toughness is better or worse, and thus we need to introduce quantitatively characterization for solution.

From the stress aspect, the film scratch failure includes not only the initiation process of micro-cracks in which the stress reaches the initial critical load, but also the extension process of micro-cracks in which cracks continue to extend as the stress increases. The failure mechanism shows that the initial critical load can only represent the crack initiation load, i.e. initial critical load reflects stage I and II; Stage III may not happens immediately if a film have good toughness. In this way, the scratch toughness ${ }^{[7,8]}$ using first critical load $\left(\mathrm{L}_{c 1}\right)$ cannot fully reflect the film toughness. ZHANG et al ${ }^{[9,10]}$ suggest that the film toughness is directly proportional to $\mathrm{L}_{c 1}$ and the difference between $\mathrm{L}_{c 1}$ and the $\mathrm{L}_{c 2}$ (second), and scratch crack propagation resistance $\left(\mathrm{CPR}_{\mathrm{S}}\right)$ can be used to characterize the toughness of a film. Since the $\mathrm{CPR}_{\mathrm{S}}$ value can reflect the film failure process, we use this value to quantitatively evaluate the film toughness.
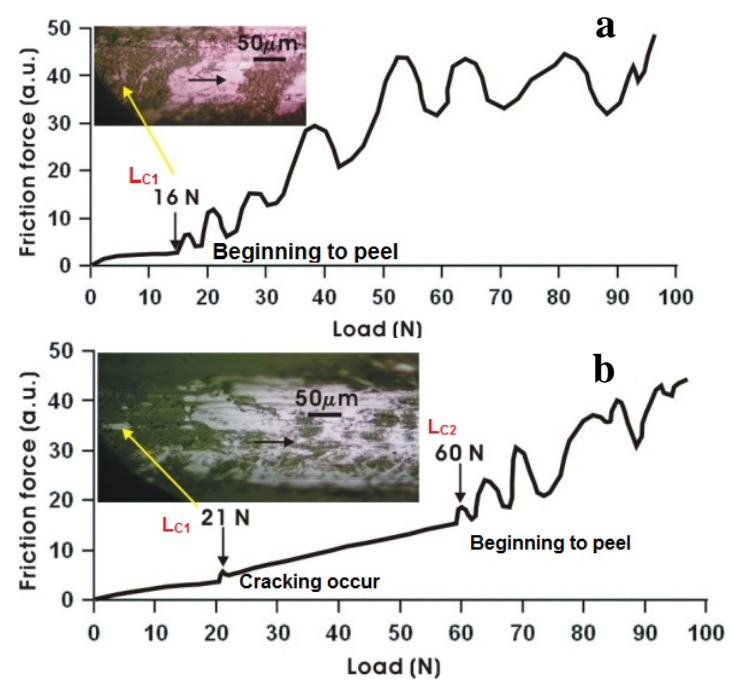

Figure 3. Critical loads of different films during the scratching process: (a) DLC film with Cr inter layer at $-100 \mathrm{~V}$; (b) DLC film with $\mathrm{Cr}$ inter layer at $-150 \mathrm{~V}$

Fig. ${ }^{[4]}$ shows our scratch result for different DLC films. The different film toughness may result in different occurrence situations of $\mathrm{L}_{c 1}$ and the $\mathrm{L}_{c 2}$. In Fig.3a, when the critical load $\left(\mathrm{L}_{c 1}\right)$ approaching to $16 \mathrm{~N}$, the microcracks occurred and extended rapidly throughout the pure DLC film, resulting in rapid film failure due to its poor toughness. Such films with poor toughness only have $L_{c 1}$. In Fig.3b, due to existence of the Cr inter layer, the DLC film exhibits better toughness than that of the pure DLC film. When the load reached $21 \mathrm{~N}\left(\mathrm{~L}_{c 1}\right)$, the film didn't deteriorate rapidly. The cracks extended at constant slow rate for a relatively long duration and began to deteriorate until the load reached $60 \mathrm{~N}\left(\mathrm{~L}_{c 2}\right)$. Such films with relatively good toughness have both $\mathrm{L}_{c 1}$ and $\mathrm{L}_{c 2}$. Based on occurrence situations of the $L_{c 1}$ and the $L_{c 2}$, the following equations can be used to calculate the $\mathrm{CPR}_{\mathrm{S}}$ of a film:

- if $\mathrm{L}_{c 2}$ doesn't occur during scratch test :

$$
\mathrm{CPR}_{\mathrm{S}}=\mathrm{L}_{c 1}{ }^{2} \text {; }
$$

- if $\mathrm{L}_{c 2}$ does occur during scratch test:

$$
\mathrm{CPR}_{\mathrm{S}}=\mathrm{L}_{c 1} *\left\{\mathrm{~K}^{*}\left(\mathrm{~L}_{c 2}-\mathrm{L}_{c 1}\right)\right\}
$$

Where, K represents a correction coefficient related to the film properties.

2) Quantitative characterization of toughness of $\mathrm{Cu}-\mathrm{DLC}$ film

We use Cu-DLC film as an example to verify feasibility of quantitative characterization of the film toughness. Fig.4 shows the results of scratch crack propagation resistance of Cu-DLC films ${ }^{[5]}$. The descending order of $\mathrm{CPR}_{\mathrm{S}}$ values (Fig.4b) for five $\mathrm{Cu}-$ DLC films is 1342.81 (a:C-Cu 10.5 at\%), 1153.62 (a:C-Cu $\mathrm{Cu}_{16.2}$ at\%), 707.94 (a:C-Cu 5.4 at\%), 580.56 (a:C-Cu $-\mathrm{Cu}_{2.7}$ at\%), and 283.02 (a:C-Cu $\mathrm{Cu}_{0}$ at\%). As Cu-DLC film with a $\mathrm{Cu}$ content of 10.5 at\% exhibits the maximal $\mathrm{L}_{c 1}, \mathrm{~L}_{c 2}-\mathrm{L}_{c 1}$, and $\mathrm{CPR}_{\mathrm{S}}$ values; we suppose that this film have an optimal toughness among the five Cu-DLC films. Its scratch morphology in Fig.4c gives us evidence for confirmation. In this way, a combination of $\mathrm{CPR}_{\mathrm{S}}$ value and scratch morphology can fully characterize the toughness of a DLC film from stress aspect. 
a

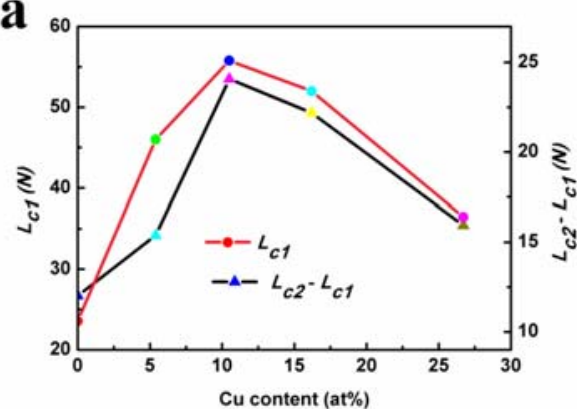

b

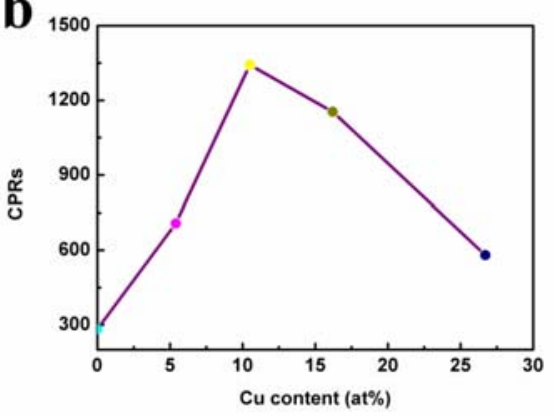

c $15 \mu \mathrm{m}$

Figure 4. Scratch test results of Cu-DLC films with different Cu contents: (a) critical loads $\left(\mathrm{L}_{C}\right)$; (b) variation curve of the CPR $\mathrm{S}_{\mathrm{S}}$ value with Cu content; (c) morphology image of the scratch on the surface of the film with a $\mathrm{Cu}$ content of 10.5 at. $\%$

\section{CONCLUSIONS}

Some interesting findings in our previous study inspire us to propose the scratch failure mechanism, so as to explore a characterization method to determine the toughness of DLC films with accuracy. The mechanism of scratch failure is suggested through analyzing the crack formation during the scratch tests of the Ag-DLC films. The failure process can be divided into three stages: (I) circular cracks formed; (II) radial cracks formed; and (III) lateral cracks formed due to crack converging. The different crack initiation and extension resistance leads to different statuses of the three stages, and brings corresponding varied scratch morphologies.

The film toughness can be qualitatively characterized by observing its scratch morphology. But for the films possess the similar scratch morphologies, we should introduce scratch crack propagation resistance $\left(\mathrm{CPR}_{\mathrm{S}}\right)$ for solution. We are safe to suppose that a combination of scratch morphology and $\mathrm{CPR}_{\mathrm{S}}$ value can accurately characterize the toughness of a DLC film from stress aspect.

\section{ACKNOWLEDGMENT}

This work was supported by the Fundamental Research Funds for Chinese Central Universities.

\section{REFERENCES}

[1] ZHAO X, HE X, SUN Y, et al. Superhard and tougher $\mathrm{SiC} /$ diamond-like-carbon composite films produced by electron beam physical vapour deposition [J]. Acta Materialia, 2009, 57 (3): 893-902.

[2] YU L D, AUKKARAVITTAYAPUN S, BOONYAWAN D, et al. Recent development in surface modification of industrial materials by ion beams and plasma at Chiang Mai University [J]. Surface and Coatings Technology, 2009, 203 (17-18): 2771-2775.

[3] YU X, NING Z W, HUA M, et al. Influence of silver incorporation on toughness improvement of diamond-Like carbon film prepared by ion beam assisted deposition [J]. The Journal of Adhesion, 2013, 89: 578-593.

[4] YU X, WANG C B, LIU Y, et al. A study of hard diamond-like carbon films in mid-frequency dual-magnetron sputtering [J]. Diamond and Related Materials, 2006, 15 (9): 1223-1227.

[5] NING Z W, YU X, HUA M, et al. Influence of copper content and nanograin size on toughness of copper containing diamond-like carbon films [J]. Materials Research Innovations, 2013, 17 (S1): 66-69.

[6] LIAO C L, GUO D, WEN S Z, et al. The Assessment of Interface Adhesion of $\mathrm{Cu} / \mathrm{Ta} /$ Black Diamond (TM)/Si Films Stack Structure by Nanoindentation and Nanoscratch Tests [J]. Tribology Letters, 2013, 53(2): 401-410.

[7] CHEN J J, BULL S J. Approaches to investigate delamination and interfacial toughness in coated systems: an overview [J]. Journal of Physics D-applied Physics, 2011, 44(3).

[8] PUJANTE J, VILASECA M, CASELlAS D, et al. High temperature scratch testing of hard PVD coatings deposited on surface treated tool steel [J]. Surface and Coatings Technology, 2014, 254: 352-357.

[9] ZHANG S, ZHANG X M. Toughness evaluation of hard coatings and thin film [J]. Thin Soild Film, 2012, 520 (7): 2375-2389.

[10] ZHANG S, SUN D, FU Y Q, et al. Effect of sputtering target power on microstructure and mechanical properties of nanocomposite nc-TiN/a-SiNx thin films [C]// 30th International Conference on Metallurgical Coatings and Thin Films. San Diego, California: Elsevier Science, 2003: 462-467. 